\title{
A LIFTING THEOREM FOR FORMAL POWER SERIES
}

\author{
EDWARD A. BENDER
}

\begin{abstract}
We define a class of operations on formal power series that includes practically all operations of interest. The class is closed under composition. We prove that an operation in this class vanishes for complex numbers if and only if it vanishes for functions analytic at the origin. A variety of applications of this "lifting theorem" are discussed.
\end{abstract}

1. Introduction. When students are uneasy about manipulating generating functions formally, they may be told something like:

Convergence doesn't matter if you're careful-most results involving convergent power series are true for formal power series because purely "algebraic" proofs can be given. Algebraic proofs for some of the basic properties of formal power series can be found in the papers by Niven [5] and Klarner [4].

In this paper, we present the basic idea behind what we consider to be a more satisfying approach: a lifting theorem is proved which implies that most identities for analytic functions hold for formal power series over any field containing the complex numbers. We illustrate the theorem by lifting a variety of results to formal power series; for example, basic arithmetic properties, elementary functions, Lagrange inversion, and roots of equations.

An identity can be thought of as a statement that some "operation" is identically zero. An operation is simply a map from formal power series to formal power series. We require that an operation possess two basic properties. Roughly speaking these are:

(A) The domain of definition of the operation $\varphi$ and the coefficients of the value of $\varphi$ are rational functions of the coefficients of the arguments of $\varphi$ (called $C$-rational).

(B) Functions analytic at the origin are mapped by $\varphi$ into functions analytic at the origin when Taylor series are used to interpret $\varphi$ for such functions (called $\boldsymbol{O}$-analytic).

These definitions will be stated precisely in the next section. The basic results are roughly:

Presented to the Society, January 25, 1973; received by the editors February 7, 1973.

AMS (MOS) subject classifications (1970). Primary 05A15, 13J05; Secondary 05A19, $32 \mathrm{~A} 05$.

(c) American Mathematical Society 1974 
I. (LIFTING Theorem). A C-rational $\mathcal{O}$-analytic operation vanishes for all formal power series over a field containing the complex numbers if and only if it vanishes for all polynomial functions.

II. (COMPOSITION THEOREM). The properties of being C-rational and $\mathcal{O}$-analytic are preserved under composition of operations.

2. Definitions. It is useful in some combinatorial applications to allow an infinite number of variables. It is also helpful to allow fields of the form $C\left(x_{1}, x_{2}, \cdots\right)$ where $C$ is the complex numbers and $x_{1}, x_{2}, \cdots$ are indeterminates. Consequently, we give the following general definition.

Definition 1. Let $F \supseteq C$ be a field, let $\omega$ be a cardinal, and let $N=$ $\{0,1,2,3, \cdots\}$. A formal power series in $\omega$ variables over $F$ is a map $f$ from the "subdirect product" $N^{[\omega]}$ to $F$. (The subdirect product $N^{[\omega]}$ consists of all $\omega$-long vectors over $N$ containing only a finite number of nonzero entries.)

We use the usual terminology and notation for the formal power series $f$ : the value of $f$ at $n$ is $f_{n}$ and is called a coefficient of the formal power series $\sum f_{n} x^{n}$, where the summation has only formal significance. (Bold face type indicates vectors.) We depart from the usual notation and denote the set of all formal power series in $\omega$ variables over $F$ by $(F, \omega)$.

It is possible to skip to $\$ 4$ now and see some typical applications. The discussion (but not the proofs) can be followed without going into the technicalities of $\$ \S 2$ and 3.

Definition 2. A function $p$ from $F^{k}$ to $F$ is a $C$-polynomial if it is a polynomial in its arguments with coefficients in $C$. A $C$-rational function is a quotient of two $C$-polynomials.

Definition 3. Let $\mathscr{S}$ and $\mathscr{T}$ be finite sets. A map $\varphi$ from $\mathscr{D}_{\varphi} \subseteq \mathscr{A}=$ $\otimes_{s \in \mathscr{S}}\left(F, \omega_{s}\right)$ into $R=\otimes_{t \in \mathscr{T}}\left(F, \omega_{t}\right)$ is called a $C$-rational operation provided

(i) every coefficient of every component of the value of $\varphi$ is a $C$ rational function of some of the coefficients of the argument of $\varphi$ and

(ii) $\mathscr{D}_{\phi}$ is the intersection of a finite number of sets of the form $\{f \in \mathscr{A}: p=0\}$ and $\{f \in \mathscr{A}: p \neq 0\}$ where $p$ is a $C$-polynomial evaluated at certain coefficients of the components of $f$. Possibly $\mathscr{D}_{\varphi}=\mathscr{A}$.

Definition 4. A function from $C^{\omega}$ to $C$ is called analytic at 0 provided it depends on only a finite number of its arguments and has a power series expansion about 0 .

The set of germs of all functions from $C^{\omega}$ to $C$ analytic at $\mathbf{0}$ is written $\mathcal{O}_{\omega}$. By an abuse of terminology we refer to germs of functions as functions. The Taylor series map $\Lambda: \mathcal{O}_{\omega} \rightarrow(C, \omega)$ is defined in the usual way by

$$
(\Lambda f)_{n}=f_{n}=\left.\left(\prod_{n_{\alpha} \neq 0} \frac{1}{n_{\alpha} !}\left(\frac{\partial}{\partial z_{\alpha}}\right)^{n_{\alpha}}\right) f(z)\right|_{z=0} .
$$


It is well known in classical analysis that $\Lambda$ is an injection and that for sufficiently small $z$

$$
f(z)=\sum f_{n} \prod_{n_{\alpha} \neq 0} z_{n_{\alpha}}^{n_{\alpha}}
$$

where the sum is over all $n \in N^{[\omega]}$ such that $f_{n} \neq 0$.

Definition 5. Let $\mathscr{B}_{\omega}$ be a subset of $\mathscr{O}_{\omega}$. A map $\varphi: \mathscr{D}_{\varphi} \rightarrow R$ is called a $\mathscr{8}$-analytic operation if

$$
\varphi: \mathscr{D}_{\varphi} \cap\left(\underset{s \in \mathscr{S}}{\otimes} \Lambda \mathscr{B}_{\omega_{s}}\right) \rightarrow \underset{t \in \mathscr{T}}{\otimes} \Lambda \mathcal{O}_{\omega_{i}}
$$

If $\varphi$ is $\mathscr{B}$-analytic, then $\Lambda^{-1} \varphi \Lambda$ maps $\mathscr{B} \cap\left(\Lambda^{-1} \mathscr{D}_{\varphi}\right)$ into $\mathcal{O}$. By an abuse of terminology we say that $\varphi$ maps $\mathscr{B} \cap\left(\Lambda^{-1} \mathscr{D}_{\varphi}\right)$ into $\mathcal{O}$.

Although $\boldsymbol{O}$-analytic $C$-rational operations are a small subset of the possible operations on formal power series, they include practically all operations of interest in enumeration.

\section{The lifting and composition theorems.}

ThEOREM 1 (LIFTING THEOREM). Let $\mathscr{P}$ denote the polynomials over $C$. If $\varphi$ is a $C$-rational $\mathscr{P}$-analytic operation which vanishes identically on the analytic functions $\left(\Lambda^{-1} \mathscr{D}_{\varphi}\right) \cap \mathscr{P}$, then $\varphi$ vanishes identically on $\mathscr{D}_{\varphi}$.

Proof. Since the assertion that $\varphi$ vanishes identically is stronger for larger fields, we can assume without loss of generality that $F$ is algebraically closed. Let the domain $\mathscr{D}_{\varphi}$ be determined by the vanishing of the $C$-polynomials $p_{i}$ and the nonvanishing of the $C$-polynomials $q_{j}$. Let a coefficient of a formal power series in the range of $\varphi$ be given by the ratio of the $C$-polynomials $r$ and $s$. It suffices to prove that

$$
\left(\forall_{i}\left(p_{i}=0\right) \wedge \forall_{j}\left(q_{j} \neq 0\right)\right) \Rightarrow(r / s=0)
$$

where the arguments of $p_{i}, q_{j}, r$ and. $s$ run over all elements of $F$. The right-hand side of (1) means $r=0$ and $s \neq 0$. We can rewrite (1) as the conjunction of the two statements

$$
\begin{aligned}
\left(\forall_{i}\left(p_{i}=0\right)\right) & \Rightarrow\left(r \prod_{j} q_{j}=0\right), \\
\left((s=0) \wedge \forall_{i}\left(p_{i}=0\right)\right) & \Rightarrow\left(\prod_{j} q_{j}=0\right) .
\end{aligned}
$$

Thus statements (2) are equivalent to the vanishing of $\varphi$ for power series over $F$. By the Hilbert Nullstellensatz [8, p. 164] equation (2a) is equivalent to the assertation that some power of $r \Pi q_{j}$ is in the ideal 
generated by the $p_{i}$ in the ring of polynomials over $F$. Since $r, q_{j}$, and $p_{i}$ are $C$-polynomials, this happens over $F$ if and only if it happens over $C$. A similar argument applies to (2b). Hence $\varphi$ vanishes identically for formal power series over $F$ if and only if (1) holds over $C$.

There exists an element of $\Lambda \mathscr{P}$ with any finite collection of coefficients specified arbitrarily. Hence (1) holds for formal power series over $C$ if and only if it holds for polynomials over $C$. Since $\varphi$ vanishes on $\left(\Lambda^{-1} \mathscr{D}_{\varphi}\right) \cap$ $\mathscr{P}$, we are done.

REMARKS. The assumption that $\varphi$ is $\mathscr{P}$-analytic is included in the theorem because it provides the link between classical analysis and formal power series. The requirement that $F \supseteq C$ is unnecessary: it is sufficient to require that $F$ have characteristic zero and $\varphi$ make sense for formal power series over $F$.

THEOREM 2 (COMPOSITION THEOREM). The composition of $\mathbf{O}$-analytic operations is $\mathbf{O}$-analytic. The composition of $C$-rational operations is C-rational.

Proof. This follows easily from the definitions.

4. Applications. A $C$-rational 0 -analytic operation which yields the same result as its analytic namesake when applied to Taylor series will be called well behaved. The theorems imply that (i) a well-behaved operation $\varphi$ vanishes on $\mathscr{D}_{\varphi}$ if and only if its namesake vanishes for polynomial functions and (ii) the composition of well-behaved operations is well behaved.

Except for division and substitution, the basic operations on formal power series are easily defined:

$$
\begin{aligned}
\left(\sum f_{n} x^{n}\right)+\left(\sum g_{n} x^{n}\right) & =\sum\left(f_{n}+g_{n}\right) x^{n}, \\
\left(\sum f_{n} x^{n}\right)\left(\sum g_{n} x^{n}\right) & =\sum h_{n} x^{n}, \\
\frac{\partial}{\partial z_{\alpha}} \sum g_{n} x^{n} & =\sum n_{\alpha} f_{n} x^{n-e_{\alpha}},
\end{aligned}
$$

etc., where $e_{x}$ is zero everywhere except for the $\alpha$ th component, which is 1 , and $h_{n}=\sum f_{k} g_{n-k}$, the sum extending over all $k$ such that $k_{\beta} \leqq n_{\beta}$ for all $\beta$. These are well-behaved operations.

To illustrate the ideas we begin with a trivial example: the formal power series over $F$ form a commutative ring with identity. Since "commutative ring with identity" can be rephrased as the vanishing of wellbehaved operations, and since $C$ is a commutative ring with identity, the lifting theorem provides a proof. As an illustration we consider commutativity in detail. It is equivalent to $f(\boldsymbol{x}) g(\boldsymbol{x})-g(\boldsymbol{x}) f(\boldsymbol{x})=0$. Define the 
operations $\psi$ and $\varphi$ by

$$
\begin{aligned}
& \psi(a, b)=(a, b, b, a)=\left(\psi_{1}, \psi_{2}, \psi_{3}, \psi_{4}\right), \\
& \varphi(a, b)=\left(\psi_{1} \psi_{2}\right)-\left(\psi_{3} \psi_{4}\right) .
\end{aligned}
$$

("Duplicating" operations like $\psi$ are quite useful in providing formal proofs.) Commutativity is equivalent to the assertion that $\varphi \equiv 0$. Since $\varphi$ is the composition of well-behaved operations, it is also well behaved. Commutativity follows from the lifting theorem. We cannot use this method to show that formal power series form an integral domain because the nonexistence of zero divisors cannot be stated as an identity. A related nonexistence result that cannot be directly lifted is the theorem that the number of solutions of an algebraic equation over an integral domain does not exceed the degree of the equation. Another sort of nonexistence result deals with general solutions of differential equations and is discussed at the end of this section.

Before discussing division it is convenient to consider substitution. Since the most general definition of substitution is not $C$-analytic, we will give a useful restricted definition. Let $x_{1}, x_{2}, \cdots, x_{k}$ be a finite number of indeterminates. Let $f, g_{1}, \cdots, g_{k}$ be formal power series over $F$ such that the coefficient of $x^{0}$ in $g_{i}$ is 0 for $1 \leqq i \leqq k$. We can then define the substitution of $x_{1}=g_{1}, \cdots, x_{k}=g_{k}$ into $f$. It is just the formula one obtains by substituting formally, expanding and collecting terms. Since this definition gives a well-behaved operation, the lifting theorem can be used in situations where substitution occurs. For example, substitution is a homomorphism for a ring of formal power series because it is a homomorphism for a ring of analytic functions.

One of the simplest examples of substitution is provided by the reciprocal. When $f_{0} \neq 0$ define

$$
1 / f(x)=f_{0}^{-1} r\left(1-f(x) / f_{0}\right)
$$

where $r(x)=1+x+x^{2}+x^{3}+\cdots$. This corresponds to the usual notion of reciprocal for analytic functions since $r(z)=1 /(1-z)$. All the usual properties of division follow from the lifting theorem.

We now turn to some less trivial applications.

By substituting into the Taylor series expansions for the elementary functions we can define these functions for formal power series. All of the traditional identities follow; for example, whenever $f_{0}=0$ and $g_{0}=0$

and

$$
e^{f(x)+g(x)}=e^{f(x)} e^{g(x)}
$$

$$
(1+f(x))^{n(x)}=\sum_{n=0}^{\infty}\left(\begin{array}{c}
h(x) \\
n
\end{array}\right) f(x)^{n}
$$


where

and

$$
\left(\begin{array}{c}
h(x) \\
n
\end{array}\right)=h(x)(h(x)-1) \cdots(h(x)+1-n) / n !
$$

$$
(1+f(x))^{h(x)}=\exp \{h(x) \log (1+f(x))\} .
$$

Let $f$ and $\boldsymbol{g}$ be $\boldsymbol{n}$ long vectors of formal power series in $\boldsymbol{n}$ indeterminates. If one writes out the formula for the $n$th coefficient of $g(f(x))$ and sets it equal to $\delta_{0 n}$, the Kronecker delta, it is easy to see that computing the inverse $g=f^{(-1)}$ of the formal power series $f(x)$ is a well-behaved operation whenever $f_{0}=0$ and the determinant $\left\|\left(\partial f_{i} / \partial x_{j}\right)_{0}\right\|$ is nonzero. See Bochner and Martin [1, p. 9] for details. Hence the properties of functional inversion can be lifted from the theory of analytic functions. The classical Lagrange inversion theorem for functions of a single variable asserts [7, p. 133]:

If $f(z)$ is analytic at $0, f_{0}=0$, and $f_{1} \neq 0$, then for sufficiently small $z$

$$
g\left(f^{(-1)}\right)(z)=g(0)+\left.\sum_{n=1}^{\infty}\left(\frac{d}{d u}\right)^{n-1}\left(g^{\prime}(u)(u / f(u))^{n}\right)\right|_{u=0} z^{n} / n ! .
$$

We have seen that functional inversion, functional composition, differentiation and multiplication are well-behaved operations. The operation $\left.\right|_{x=0}$ defined by $\left(\left.f\right|_{x=0}\right)_{n}=f_{0} \delta_{0 n}$ is clearly a well-behaved operation. What about $u / f(u)$ ? Since $f_{0}=0$, the reciprocal of $f$ is not defined. On the other hand, we can take the reciprocal of the formal power series $h$ defined by $h_{n}=f_{n+1}$. This is a well-behaved operation corresponding to $1 /(f(z) / z)$. Finally, the infinite sum can be viewed simply as a notation for a formal power series. Since both sides of the Lagrange inversion formula can be interpreted as well-behaved operations, the formula is valid for formal power series. An algebraic proof is given by Henrici [3] and a combinatorial proof by Raney [6]. The multivariate form of Lagrange inversion (Good [2, Theorem 12]) can also be lifted.

Formulas for solutions of equations can often be interpreted as wellbehaved operations to which the lifting theorem can be applied. In general it is not true that all solutions are obtained in this way. The following examples illustrate some problems:

(i) The algebraic equation $f^{2}=x$ has no formal power series solution.

(ii) The formal power series $g(x)=\sum(n-1) ! x^{n}$ satisfies the differential equation $x^{2} g^{\prime}=g-x$. The analytic solution

$$
g(x)=-e^{-1 / x} \int x^{-1} e^{1 / x} d x
$$

involves a function which is not analytic at 0 . 
We will consider ordinary differential equations in a bit more detail. It is easy to provide arbitrary constants: use the operation $\left.\right|_{x=0}$ defined previously. To prove that a lifted solution is the general solution it suffices to show that (i) the $m$ arbitrary constants allow the first $m$ coefficients of the solution to be specified arbitrarily and (ii) the remaining coefficients are determined recursively by the differential equation. If the equation can be put in the form $y^{(m)}=h\left(x, y, y^{\prime}, y^{\prime \prime}, \cdots, y^{(m-1)}\right)$ then the argument for analytic functions (i.e., equate coefficients of $x^{n}$ ) can be used.

To illustrate these ideas we consider the general solution of the equation $g^{\prime}(x)=g(x) c^{\prime}(x)$ which arises in the enumeration of labeled graphs. The field of coefficients for $g(x)$ and $c(x)$ is $C\left(x_{1}, x_{2}, \cdots\right)$. The general solution for analytic functions is $g(x)=e^{c(x)+k}$ where $k$ is an arbitrary constant. In general this cannot be lifted because we have only defined the exponential function for power series without a constant term. Therefore we rewrite the solution in the form $g(x)=K e^{c(x)-c_{0}}$ where $K$ is an arbitrary constant. This can be lifted to formal power series. Note that $g_{0}$ is completely arbitrary. Since this is precisely what is needed for a general solution, this solution is general.

5. The quotient field. It is sometimes useful to deal with the quotient field of the ring of formal power series over $F$. The above results can be carried over to this situation by replacing references to $\mathcal{O}$ by references to functions with finite poles at 0 , references to $\mathscr{P}$ by references to functions with finite Laurent expansions about 0 , and the phrase "is analytic (at $\mathbf{0}$ )" by the phrase "is analytic or has a pole (at $\mathbf{0})$."

\section{REFERENCES}

1. S. Bochner and W. T. Martin, Several complex variables, Princeton Math. Series, vol. 10, Princeton Univ. Press, Princeton, N.J., 1948. MR 10, 366.

2. I. J. Good, Generalizations to several variables of Lagrange's expansion, with applications to stochastic processes, Proc. Cambridge Phil. Soc. 56 (1960), 367-380. MR 23 \#A352.

3. P. Henrici, An algebraic proof of the Lagrange-Bürmann formula, J. Math. Anal. Appl. 8 (1964), 218-224. MR 28 \#2198.

4. D. A. Klarner, Algebraic theory for difference and differential equations, Amer. Math. Monthly 76 (1969), 366-373. MR 39 \#3252.

5. I. Niven, Formal power series, Amer. Math. Monthly 76 (1969), 871-889. MR 40 \#5606.

6. G. Raney, Functional composition patterns and power series reversion, Trans. Amer. Math. Soc. 94 (1960), 441-451. MR 22 \#5584.

7. E. T. Whittaker and G. N. Watson, $A$ course of modern analysis 4th ed. (reprint), Cambridge Univ. Press, Cambridge, 1962. MR 31 \#2375.

8. O. Zariski and P. Samuel, Commutative algebra. Vol. II, University Series in Higher Math., Van Nostrand, Princeton, N.J., 1960. MR 22 \#11006.

Institute for Defense ANAlyses, Princeton, New Jersey 08540 